Spectral analysis of saddle point matrices with indefinite leading blocks

N I M Gould V Simoncini

22nd August 2008 


\section{(c) Science and Technology Facilities Council}

Enquires about copyright, reproduction and requests for additional copies of this report should be addressed to:

Library and Information Services

SFTC Rutherford Appleton Laboratory

Harwell Science and Innovation Campus

Didcot

OX11 OQX

UK

Tel: $+44(0) 1235445384$

Fax: $+44(0) 1235446403$

Email: library@rl.ac.uk

The STFC ePublication archive (epubs), recording the scientific output of the Chilbolton, Daresbury, and Rutherford Appleton Laboratories is available online at: http://epubs.cclrc.ac.uk/

ISSN 1358-6254

Neither the Council nor the Laboratory accept any responsibility for loss or damage arising from the use of information contained in any of their reports or in any communication about their tests or investigation 


\title{
Spectral analysis of saddle point matrices with indefinite leading blocks
}

\author{
Nicholas I. M. Gould ${ }^{1,2}$ and Valeria Simoncini ${ }^{3}$
}

\begin{abstract}
We provide eigenvalue intervals for symmetric saddle-point and regularised saddle-point matrices in the case where the $(1,1)$ block may be indefinite. These generalise known results for the definite $(1,1)$ case. We also study the spectral properties of the equivalent augmented formulation, which is an alternative to explicitly dealing with the indefinite $(1,1)$ block. Such an analysis may be used to assess the convergence of suitable Krylov subspace methods. We conclude with spectral analyses of the effects of common block-diagonal preconditioners.
\end{abstract}

1 This work was supported by the EPSRC grants EP/E053351/1 and EP/F005369/1.

${ }^{2}$ Computational Science and Engineering Department, Rutherford Appleton Laboratory, Chilton, Oxfordshire, OX11 0QX, England, EU.

Email: n.i.m.gould@rl.ac.uk .

Current reports available from "http://www.numerical.rl.ac.uk/reports/reports.shtml".

${ }^{3}$ Dipartimento di Matematica, Università di Bologna, Piazza di Porta S. Donato 5,

I-40127 Bologna, Italy, EU and CIRSA, Ravenna, Italy, EU.

Email: valeria@dm.unibo.it .

Current reports available from "http://www.dm.unibo.it/ simoncin/list.html".

Computational Science and Engineering Department

Atlas Centre

Rutherford Appleton Laboratory

Oxfordshire OX11 0QX

22nd August 2008 


\section{Introduction}

We consider the matrix

$$
\mathcal{M}=\left[\begin{array}{cc}
A & B^{T} \\
B & 0
\end{array}\right]
$$

We assume that $B$ is $m$ by $n(m \leq n)$ and of full row rank, that $A$ is symmetric and indefinite, and that $A$ is positive definite on the kernel of $B$. These hypotheses imply that the matrix $\mathcal{M}$ is nonsingular. We also consider the "stabilized" matrix

$$
\mathcal{M}_{C}=\left[\begin{array}{cc}
A & B^{T} \\
B & -C
\end{array}\right]
$$

where in addition to the hypotheses of $A$ and $B$, we require that $C$ be symmetric positive semidefinite.

Problems involving matrices of the form $\mathcal{M}$ for which $A$ is symmetric but indefinite occur throughout (equality-)constrained nonlinear optimization - in this field $\mathcal{M}$ is known as the Karush-Kuhn-Tucker (KKT) matrix [13, §16.1]. To be specific, SQP methods for finding the smallest value of the objective function $f(x)$ where $x$ is constrained to satisfy $c(x)=0$ build a correction $s$ from a current estimate $x$ by minimizing a quadratic approximation $g(x)^{T} s+\frac{1}{2} s^{T} A s$ of the related Lagrangian function subject to a linearization $B(x) s+c(x)=0$ of the constraints $[13, \S 18.1]$. Here $g$ is the gradient of $f, A$ represents the Hessian of a Lagrangian function, while $B$ is the Jacobian of the constraints. This directionfinding subproblem should only be solved if $\mathcal{M}$ has "correct" inertia-specifically that $A$ be sufficiently positive definite on the null-space of $B$ [13, Lem.16.1-Thm.16.3] — and in this case the desired correction $s$ and an associate vector of Lagrange multipliers $y$ satisfy the linear system (where for brevity we have dropped the argument $x$ in the right-hand side functions)

$$
\left[\begin{array}{cc}
A & B^{T} \\
B & 0
\end{array}\right]\left[\begin{array}{l}
s \\
y
\end{array}\right]=-\left[\begin{array}{l}
g \\
c
\end{array}\right]
$$

The assumption that the Hessian should be positive definite on the null-space of the constraints is a second-order sufficiency condition for a constrained optima [13, Thm.12.6]. Note that this requirement is in contrast to superficially-similar systems from CFD where it is often natural to assume that $A$ itself is positive (semi-) definite $[6, \S 5.5]$.

Problems involving $\mathcal{M}_{C}$ with indefinite $A$ but semidefinite $C$ arise when solving inequality-constrained nonlinear optimization problems using penalty or interior-point methods ( $C$ positive definite) or with a mix of equality and inequality constraints $(C$ positive semidefinite but singular). Specifically, interior-point methods for minimizing an objective $f(x)$ constrained by inequalities $c(x) \geq 0$ transform the problem into a sequence of unconstrained optimizations of "barrier" functions $\phi(x, \nu)=f(x)+\nu \Phi(c(x))$, where the parameter $\nu \rightarrow 0_{+}$and $\Phi$ is finite at strictly feasible $x$ but infinite elsewhere [13, §19.1]. To solve each barrier problem, a correction $s$ from a current estimate $x$ is determined by minimizing the quadratic Taylor approximation $s^{T} \nabla_{x} \phi+\frac{1}{2} s^{T} \nabla_{x x} \phi s$. This problem should 
only be solved if the Hessian $\nabla_{x x} \phi$ is positive definite, and in this case $s$ satisfies the Newton equations

$$
\nabla_{x x} \phi s=-\nabla_{x} \phi
$$

Generically $\nabla_{x} \phi$ and $\nabla_{x x} \phi$ have the form $g+J^{T} e$ and $H+J^{T} D J$ respectively, where $H$ is the Hessian of the Lagrangian, $g$ is the gradient of $f, e$ is a vector of Lagrange multiplier estimates, $J$ is the constraint Jacobian and $D$ is a diagonal matrix of positive weights some of which converge to zero while the others diverge. Thus $\nabla_{x x} \phi$ is almost invariably ill conditioned, and it may be preferable to solve the equivalent

$$
\left[\begin{array}{cc}
H+J_{0}^{T} D_{0} J_{0} & J_{\infty}^{T} \\
J_{\infty} & -D_{\infty}^{-1}
\end{array}\right]\left[\begin{array}{l}
s \\
v
\end{array}\right]=-\left[\begin{array}{c}
g+J_{0}^{T} e_{0} \\
e_{\infty}
\end{array}\right]
$$

involving some auxiliary $v$, where the subscripts 0 and $\infty$ refer to the partition of indices for the components of $D$ which converge and diverge respectively, rather than (1.1) [12, $\S 6]$. Note that there is reason to expect $H$ to be positive definite, but necessarily it will be so on the null-space of $J_{\infty}$ whenever $\nabla_{x x} \phi$ is positive definite. Thus the coefficient matrix of (1.2) is of the form $\mathcal{M}_{\mathcal{C}}$ with a positive definite $C$, and although $C \neq 0$, we do expect in general that $\|C\|$ will be small. It is also most likely in practice that at most $m \leq n$ of the (diagonal) components in $D$ diverge. ${ }^{1}$

If in addition we have explicit equality constraints $c_{E}(x)=0$ with Jacobian $J_{E}$, then just as in $\S 1,(1.2)$ becomes

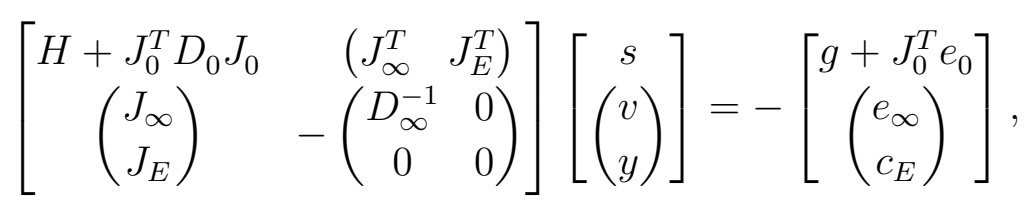

which again has a coefficient matrix of the form $\mathcal{M}_{\mathcal{C}}$, but $C$ is now only positive semidefinite. Once again, it is most likely in practice that $m \leq n$ from such applications if they have been properly reformulated.

Another interesting case arises when $A$ itself has (recursive) saddle-point structure [10] and is thus indefinite.

We note that superficially-similar systems from CFD, specifically from stabilised Stokes flow, often naturally assume that $A$ is positive (semi-) definite $[6, \S 5.3 .2]$.

Our purpose here is to understand how weakening the requirement that $A$ be everywhere definite to simply definite in a subspace influences the bounds we may deduce on the eigenvalues of $\mathcal{M}$ and of $\mathcal{M}_{C}$. This is important since quantitative understanding of the convergence of symmetric iterative methods like MINRES for generic linear systems

$$
\left[\begin{array}{cc}
A & B^{T} \\
B & 0
\end{array}\right]\left[\begin{array}{l}
x \\
y
\end{array}\right]=\left[\begin{array}{l}
a \\
b
\end{array}\right] \text { and }\left[\begin{array}{cc}
A & B^{T} \\
B & -C
\end{array}\right]\left[\begin{array}{l}
x \\
y
\end{array}\right]=\left[\begin{array}{l}
a \\
b
\end{array}\right]
$$

\footnotetext{
${ }^{1}$ Diverging components correspond to strictly complementary asymptotically active constraints $[12, \S 6]$.
} 
is governed by the knowledge of the eigenvalues of the system matrix [6, Thm.6.13]. In particular, when (as is the case here) the matrix is indefinite and the eigenvalues lie in intervals $[a, b] \cup[c, d]$ with $a \leq b<0<c \leq d$, better convergence estimates are possible than by simply knowing the matrix's condition number. Realistic eigenvalue intervals when $A$ is definite are often available [15, Lem.2.1] — see the survey article [3] for more detailsbut our aim is to present similar results in our more-general context. We also analyze the equivalent augmented formulation, where the original linear system is transformed so as to make the $(1,1)$ block definite. We explicitly show how the spectral properties of the resulting matrix depend on the augmentation parameter, and provide a sufficient condition on the parameter so that the $(1,1)$ block is indeed positive definite.

The paper is organized as follows. In Section 2 we provide estimates for the real intervals containing the spectrum of $\mathcal{M}$, while in Section 2.1 we refine our estimates by including additional knowledge on the data. In Section 2.2 we derive spectral bounds when the original problem with $\mathcal{M}$ is replaced by an equivalent augmented formulation. In Section 3 we derive interval estimates for the spectrum of $\mathcal{M}_{C}$. In section 4 we briefly discuss natural generalizations of a known class of preconditioners.

\section{Estimates for the eigenvalues of $\mathcal{M}$}

Here we analyze the location of the spectrum of $\mathcal{M}$.

We start with a general inertia-characterization result - the inertia of a matrix $M$, $\operatorname{In}(M)$, is the triple $\left(m_{+}, m_{-}, m_{0}\right)$, where $m_{+}, m_{-}$and $m_{0}$ are the numbers of positive, negative and zero eigenvalues of $M$, respectively.

Proposition 2.1. [4, 11]. Suppose that the columns of $Z$ provide an orthonormal basis for the null-space of $A$. Then $\operatorname{In}(\mathcal{M})=\operatorname{In}\left(Z^{T} A Z\right)+(m, m, 0)$.

Now assume that $A$ is positive definite on the kernel of $B$. In this case, $Z^{T} A Z$ is positive definite, and Proposition 2.1 shows that $\mathcal{M}$ has precisely $n$ positive and $m$ negative eigenvalues. We note that the leftmost eigenvalue of $Z^{T} A Z$ satisfies

$$
\lambda_{\min }^{\mathrm{z}}=\min _{0 \neq x \in \mathcal{N}(B)} \frac{x^{T} A x}{x^{T} x} .
$$

Proposition 2.2. Assume that $A$ is positive definite on the kernel of $B$, so that the leftmost eigenvalue of $Z^{T} A Z, \lambda_{\min }^{Z}>0$. Let $\lambda_{\min }^{A}, \lambda_{\max }^{A}$ be the leftmost (perhaps negative) and rightmost eigenvalues of $A$, and let $\sigma_{\min }, \sigma_{\max }$ be the smallest nonzero and largest singular values of the full rank matrix $B$. Then the eigenvalues $\mu$ of $\mathcal{M}$ are contained in $\mathcal{I}^{-} \cup \mathcal{I}^{+} \subset \mathbb{R}$ with

$$
\begin{aligned}
& \mathcal{I}^{-}=\left[\frac{1}{2}\left(\lambda_{\text {min }}^{A}-\sqrt{\left(\lambda_{\text {min }}^{A}\right)^{2}+4 \sigma_{\text {max }}^{2}}\right), \frac{1}{2}\left(\lambda_{\text {max }}^{A}-\sqrt{\left(\lambda_{\max }^{A}\right)^{2}+4 \sigma_{\text {min }}^{2}}\right)\right] \subset \mathbb{R}^{-} \\
& \mathcal{I}^{+}=\left[\gamma, \frac{1}{2}\left(\lambda_{\text {max }}^{A}+\sqrt{\left(\lambda_{\text {max }}^{A}\right)^{2}+4 \sigma_{\max }^{2}}\right)\right] \subset \mathbb{R}^{+}
\end{aligned}
$$


where $\gamma<\lambda_{\min }^{Z}$ is the smallest positive root of the cubic equation

$$
\mu^{3}-\mu^{2}\left(\lambda_{\min }^{Z}+\lambda_{\min }^{A}\right)+\mu\left(\lambda_{\min }^{Z} \lambda_{\min }^{A}-\|A\|^{2}-\sigma_{\min }^{2}\right)+\lambda_{\min }^{Z} \sigma_{\min }^{2}=0 .
$$

In particular,

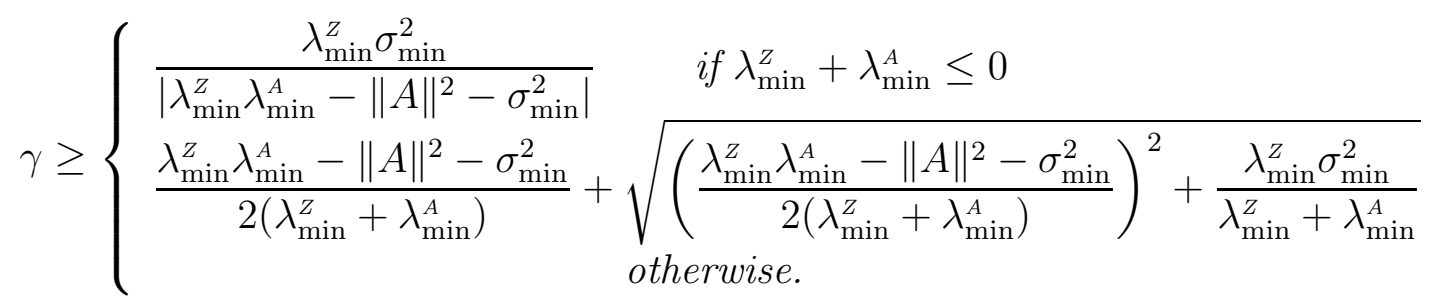

If $m=n$, we have instead

$$
\mathcal{I}^{+}=\left[\frac{1}{2}\left(\lambda_{\text {min }}^{A}+\sqrt{\left(\lambda_{\text {min }}^{A}\right)^{2}+4 \sigma_{\min }^{2}}\right), \frac{1}{2}\left(\lambda_{\max }^{A}+\sqrt{\left(\lambda_{\max }^{A}\right)^{2}+4 \sigma_{\max }^{2}}\right)\right] \subset \mathbb{R}^{+} .
$$

Proof. Let $[x ; y]$ be an eigenvector corresponding to an eigenvalue $\mu$. We first consider the case that $y=0$. Then $A x=\mu x$ and $B x=0$, so that $\lambda_{\text {min }}^{\mathrm{z}} \leq \mu \leq \lambda_{\max }^{\mathrm{A}}$. The same is true if $B x=0$, as then $y=0$. Furthermore since $B$ is full rank, $x \neq 0$, since otherwise this would also imply $y=0$. So henceforth, we assume that none of these special cases occur.

We proceed by evaluating the extremes of $\mathcal{I}^{-}$. For $\mu \neq 0$, substituting the second equation into the first one and reordering terms yields

$$
\mu^{2} x-\mu A x-B^{T} B x=0 .
$$

Multiplying by the left by $x^{T}$ we obtain

$$
\mu^{2}\|x\|^{2}-\mu x^{T} A x-\|B x\|^{2}=0 .
$$

Since $\mu<0$, we have $-\mu x^{T} A x \geq-\mu \lambda_{\min }^{\mathrm{A}}\|x\|^{2}$. Using also $-\|B x\|^{2} \geq-\sigma_{\max }^{2}\|x\|^{2}$ we get the inequality

$$
\mu^{2}-\mu \lambda_{\min }^{\mathrm{A}}-\sigma_{\max }^{2} \leq 0,
$$

from which the left extreme follows. To proceed with the right extreme of $\mathcal{I}^{-}$we let $x=x_{0}+x_{1}$, with $x_{0} \in \mathcal{N}(B)$ and $0 \neq x_{1} \in \operatorname{Range}\left(B^{T}\right)$. We multiply the equation (2.5) by $x_{1}^{T}$ and by $x_{0}^{T}$. Using the fact that $B x=B x_{1}$, we obtain

$$
\begin{aligned}
\mu^{2}\left\|x_{1}\right\|^{2}-\mu x_{1}^{T} A x_{1}-\mu x_{1}^{T} A x_{0}-\left\|B x_{1}\right\|^{2} & =0 \\
\mu^{2}\left\|x_{0}\right\|^{2}-\mu x_{0}^{T} A x_{1}-\mu x_{0}^{T} A x_{0} & =0 .
\end{aligned}
$$

Subtracting the second from the first equation and recalling that $\mu<0$ and $x_{0}^{T} A x_{0}>0$ we get

$$
\begin{aligned}
0 & =\mu^{2}\left\|x_{1}\right\|^{2}-\mu^{2}\left\|x_{0}\right\|^{2}+\mu x_{0}^{T} A x_{0}-\mu x_{1}^{T} A x_{1}-\left\|B x_{1}\right\|^{2} \\
& \leq \mu^{2}\left\|x_{1}\right\|^{2}-\mu x_{1}^{T} A x_{1}-\left\|B x_{1}\right\|^{2} \\
& \leq\left(\mu^{2}-\mu \lambda_{\max }^{\mathrm{A}}-\sigma_{\min }^{2}\right)\left\|x_{1}\right\|^{2} .
\end{aligned}
$$


For $x_{1} \neq 0$ the required negative upper bound follows.

We next determine the extremes of $\mathcal{I}^{+}$keeping in mind that $\mu>0$. We use (2.8) and the facts that $x^{T} A x \leq \lambda_{\max }^{\mathrm{A}}\|x\|^{2}$ and $\|B x\| \leq \sigma_{\max }\|x\|$ to get $\mu^{2}-\mu \lambda_{\max }^{\mathrm{A}}-\sigma_{\max }^{2} \leq 0$, from which the stated upper bound follows. When $m=n$, we also have $\lambda_{\min }^{\mathrm{A}}\|x\|^{2} \leq x^{T} A x$ and $\sigma_{\min }\|x\| \leq\|B x\|$ which with $(2.8)$ give $\mu^{2}-\mu \lambda_{\text {min }}^{\mathrm{A}}-\sigma_{\min }^{2} \geq 0$, from which the left bound results.

When $m<n$, the left bound, $\gamma$, is more delicate. We write $x=x_{0}+x_{1}$ as before. We next assume that $\mu<\lambda_{\min }^{z}$, otherwise $\lambda_{\text {min }}^{z}$ is the sought-after extreme. From equation (2.8) we see that

$$
x_{0}^{T} A x_{1}=\mu\left\|x_{0}\right\|^{2}-x_{0}^{T} A x_{0} \leq\left(\mu-\lambda_{\min }^{\mathrm{z}}\right)\left\|x_{0}\right\|^{2}, \quad x_{0}^{T} A x_{1} \geq-\|A\|\left\|x_{1}\right\|\left\|x_{0}\right\| .
$$

Using $\mu-\lambda_{\min }^{\mathrm{z}}<0$, the two inequalities above also imply that $\left\|x_{0}\right\| \leq-\|A\|\left\|x_{1}\right\| /(\mu-$ $\left.\lambda_{\text {min }}^{\mathrm{z}}\right)$, so that $-x_{0}^{T} A x_{1} \leq\|A\|\left\|x_{1}\right\|\left\|x_{0}\right\| \leq-\|A\|^{2}\left\|x_{1}\right\|^{2} /\left(\mu-\lambda_{\min }^{\mathrm{z}}\right)$. Therefore, we can bound (2.7) as

$$
\begin{aligned}
0 & =\mu^{2}\left\|x_{1}\right\|^{2}-\mu x_{1}^{T} A x_{1}-\mu x_{1}^{T} A x_{0}-\left\|B x_{1}\right\|^{2} \\
& \leq\left(\mu^{2}-\mu \lambda_{\min }^{\mathrm{A}}+\mu\|A\|^{2} /\left(\lambda_{\min }^{\mathrm{z}}-\mu\right)-\sigma_{\min }^{2}\right)\left\|x_{1}\right\|^{2},
\end{aligned}
$$

which, as $x_{1} \neq 0$, is equivalent to

$$
\phi(\mu) \equiv \mu^{3}-\mu^{2}\left(\lambda_{\min }^{\mathrm{z}}+\lambda_{\min }^{\mathrm{A}}\right)+\mu\left(\lambda_{\min }^{\mathrm{z}} \lambda_{\min }^{\mathrm{A}}-\|A\|^{2}-\sigma_{\min }^{2}\right)+\lambda_{\min }^{\mathrm{z}} \sigma_{\min }^{2} \leq 0 .
$$

Note that $\phi\left(\lambda_{\text {min }}^{\mathrm{z}}\right)=-\lambda_{\text {min }}^{\mathrm{z}}\|A\|^{2}<0$, and thus the required bound $\gamma<\lambda_{\min }^{\mathrm{z}}$. Since $\mu^{3}>0$ we bound the left expression from below as

$$
-\mu^{2}\left(\lambda_{\min }^{\mathrm{Z}}+\lambda_{\min }^{\mathrm{A}}\right)+\mu\left(\lambda_{\min }^{\mathrm{Z}} \lambda_{\min }^{\mathrm{A}}-\|A\|^{2}-\sigma_{\min }^{2}\right)+\lambda_{\min }^{\mathrm{Z}} \sigma_{\min }^{2} \leq 0 .
$$

If $\lambda_{\min }^{\mathrm{z}}+\lambda_{\min }^{\mathrm{A}}>0$ then we rewrite

$$
\mu^{2}\left(\lambda_{\min }^{\mathrm{Z}}+\lambda_{\min }^{\mathrm{A}}\right)-\mu\left(\lambda_{\min }^{\mathrm{Z}} \lambda_{\min }^{\mathrm{A}}-\|A\|^{2}-\sigma_{\min }^{2}\right)-\lambda_{\min }^{\mathrm{Z}} \sigma_{\min }^{2} \geq 0,
$$

from which the stated value of $\gamma$ follows. If conversely $\lambda_{\min }^{\mathrm{Z}}+\lambda_{\min }^{\mathrm{A}} \leq 0$ then $\mu^{2}\left(\lambda_{\min }^{\mathrm{z}}+\right.$ $\left.\lambda_{\min }^{\mathrm{A}}\right) \leq 0$ and we obtain

$$
\mu\left(\lambda_{\min }^{\mathrm{Z}} \lambda_{\min }^{\mathrm{A}}-\|A\|^{2}-\sigma_{\min }^{2}\right)+\lambda_{\min }^{\mathrm{Z}} \sigma_{\min }^{2} \leq 0 .
$$

Since $\lambda_{\min }^{\mathrm{Z}} \lambda_{\min }^{\mathrm{A}}-\|A\|^{2}-\sigma_{\min }^{2} \leq 0$, the value of $\gamma$ for $\lambda_{\min }^{\mathrm{Z}}+\lambda_{\min }^{\mathrm{A}} \leq 0$ follows.

By way of contrast, when $A$ is positive definite the following result is known.

Proposition 2.3. [15, Lem.2.1] Assume that $A$ is positive definite, let $\lambda_{\min }^{A}, \lambda_{\max }^{A}$ be the smallest (positive) and largest (positive) eigenvalues of $A$, and let $\sigma_{\min }, \sigma_{\max }$ be the smallest nonzero and largest singular values of the full rank matrix $B$. Then the eigenvalues $\mu$ of $\mathcal{M}$ are contained in $\mathcal{I}^{-} \cup \mathcal{I}^{+} \subset \mathbb{R}$ with

$$
\begin{aligned}
& \mathcal{I}^{-}=\left[\frac{1}{2}\left(\lambda_{\text {min }}^{A}-\sqrt{\left(\lambda_{\text {min }}^{A}\right)^{2}+4 \sigma_{\text {max }}^{2}}\right), \frac{1}{2}\left(\lambda_{\text {max }}^{A}-\sqrt{\left(\lambda_{\text {max }}^{A}\right)^{2}+4 \sigma_{\text {min }}^{2}}\right)\right] \subset \mathbb{R}^{-} \\
& \mathcal{I}^{+}=\left[\lambda_{\text {min }}^{A}, \frac{1}{2}\left(\lambda_{\text {max }}^{A}+\sqrt{\left(\lambda_{\text {max }}^{A}\right)^{2}+4 \sigma_{\text {max }}^{2}}\right)\right] \subset \mathbb{R}^{+} .
\end{aligned}
$$


We see that the only difference in the positive definite and indefinite cases is how the smallest positive eigenvalue of $\mathcal{M}$ is constrained by that of $A$ in the former case, while it is the interaction between that of $A$ and $Z^{T} A Z$ which plays a role in the latter.

The bounds in Proposition 2.2 appear to be quite sharp, as shown by the following examples (all reported computed numbers are exact to the first few decimal digits).

Example 2.4. We consider the matrices

$$
A=\left[\begin{array}{rr}
1 & -3 \\
-3 & 2
\end{array}\right], \quad B^{T}=\left[\begin{array}{l}
0 \\
0.1
\end{array}\right]
$$

The corresponding matrix $\mathcal{M}$ has eigenvalues

$$
\mu_{1}=-1.5441, \quad \mu_{2}=0.0014257, \quad \mu_{3}=4.5427,
$$

which belong to the intervals obtained in Proposition 2.2, cf. Table 2.1. Note in particular the sharpness of the right extreme of $\mathcal{I}^{+}$and of the left extreme of $\mathcal{I}^{-}$.

Example 2.5. We consider the matrices

$$
A=\left[\begin{array}{rr}
0.1 & 2 \\
2 & 0.1
\end{array}\right], \quad B^{T}=\left[\begin{array}{l}
0 \\
1
\end{array}\right]
$$

Then $\mu(\mathcal{M})=\{-2.1465,0.020026,2.3264\}$, while the bounding intervals are reported in Table 2.1. Note the sharpness of the left extreme of $\mathcal{I}^{+}$.

Example 2.6. We consider the matrices

$$
A=\left[\begin{array}{rr}
0.01 & 3 \\
3 & -0.01
\end{array}\right], B=[0,3]
$$

In this case, $\mu(\mathcal{M})=\left\{-4.2452,5.0 \cdot 10^{-3}, 4.2402\right\}$. Note once again the sharpness of the left extreme of $\mathcal{I}^{+}$.

Example 2.7. We consider the matrices

$$
A=\left[\begin{array}{rrr}
1 & -4 & 0 \\
-4 & -1 & 0 \\
0 & 0 & 2
\end{array}\right], \quad B^{T}=\left[\begin{array}{ll}
0 & 1 \\
1 & 0 \\
0 & 0
\end{array}\right]
$$

Then $\mu(\mathcal{M})=\{-4.3528,-0.22974,0.22974,2,4.3528\}$, while the bounding intervals are reported in Table 2.1. Note the sharpness of both extremes of $\mathcal{I}^{-}$and of the right extreme of $\mathcal{I}^{+}$. 


\begin{tabular}{|l|c|c|c|c|c|c|}
\hline case & $\lambda_{\min }^{\mathrm{A}}$ & $\lambda_{\max }^{\mathrm{A}}$ & $\lambda_{\min }^{\mathrm{Z}}$ & $\sigma_{\min }, \sigma_{\max }$ & $\mathcal{I}^{-}$ & $\mathcal{I}^{+}$ \\
\hline Ex. 2.4 & -1.5414 & 4.5414 & 1.0 & $0.1,0.1$ & {$[-1.5478,-0.0022]$} & {$[0.0004,4.5436]$} \\
Ex. 2.5 & -1.9000 & 2.1000 & 0.1 & 1,1 & {$[-2.3293,-0.40000]$} & {$[0.017857,2.50]$} \\
Ex. 2.6 & -3.0000 & 3.0000 & 0.01 & 3,3 & {$[-4.8541,-1.8541]$} & {$\left[4.9917 \cdot 10^{-3}, 4.8541\right]$} \\
Ex. 2.7 & -4.1231 & 4.1231 & 2.0 & 1,1 & {$[-4.3528,-0.22974]$} & {$[0.0762,4.3528]$} \\
\hline
\end{tabular}

Table 2.1: Spectral information and bounds for the examples in section 2.

\section{$2.1 \quad$ Specialized results}

We can improve on the eigenvalue bounds given in Proposition 2.2 if we make further assumptions on the data. Recall that we are assuming that $B$ is of full rank. In this case, we may decompose $B(Y Z)=(L 0)$, where $(Y Z)$ is orthogonal and $L$ is nonsingular - an LQ factorization is one possibility. Note that $B$ and $L$ have the same (nonzero) singular values. In this case, $\mathcal{M}$ is similar to

$$
\left[\begin{array}{ccc}
Z^{T} A Z & Z^{T} A Y & 0 \\
Y^{T} A Z & Y^{T} A Y & L^{T} \\
0 & L & 0
\end{array}\right] .
$$

This leads directly to the following result.

Proposition 2.8. Let $\sigma_{\min }, \sigma_{\max }$ be the smallest nonzero and largest singular values of the full rank matrix $B$, and suppose that $B\left(\begin{array}{l}Y \\ Z\end{array}\right)=\left(\begin{array}{ll}L & 0\end{array}\right)$, where $\left(\begin{array}{l}Y Z \\ Y\end{array}\right)$ is orthogonal and $L$ is nonsingular. Suppose further that $Z^{T} A Z$ has extreme eigenvalues $\lambda_{\min }^{Z} \leq \lambda_{\max }^{Z}$, that $Y^{T} A Y$ has extreme eigenvalues $\lambda_{\min }^{Y} \leq \lambda_{\max }^{Y}$ and that $Z^{T} A Y=0$. Then the eigenvalues $\mu$ of $\mathcal{M}$ are contained in

$$
\begin{aligned}
& {\left[\frac{1}{2}\left(\lambda_{\min }^{Y}-\sqrt{\left(\lambda_{\min }^{Y}\right)^{2}+4 \sigma_{\max }^{2}}\right), \frac{1}{2}\left(\lambda_{\max }^{Y}-\sqrt{\left(\lambda_{\max }^{Y}\right)^{2}+4 \sigma_{\min }^{2}}\right)\right] } \\
\cup & {\left[\frac{1}{2}\left(\lambda_{\min }^{Y}+\sqrt{\left(\lambda_{\min }^{Y}\right)^{2}+4 \sigma_{\min }^{2}}\right), \frac{1}{2}\left(\lambda_{\max }^{Y}+\sqrt{\left(\lambda_{\max }^{Y}\right)^{2}+4 \sigma_{\max }^{2}}\right)\right] } \\
\cup & {\left[\lambda_{\min }^{Z}, \lambda_{\max }^{Z}\right] . }
\end{aligned}
$$

Proof. Since $\mathcal{M}$ is similar to (2.9) and as by assumption $Z^{T} A Y=0$, it follows immediately that $n-m$ of the eigenvalues of $\mathcal{M}$ are those of $Z^{T} A Z$, while the remainder are those of

$$
\left[\begin{array}{cc}
Y^{T} A Y & L^{T} \\
L & 0
\end{array}\right]
$$

Applying Proposition 2.2 to (2.10) for the square (" $m=n$ ") case gives the remaining eigenvalue intervals.

If we drop the assumption that $Z^{T} A Y=0$, we may derive slightly weaker bounds. 
Proposition 2.9. Let $\sigma_{\min }, \sigma_{\max }$ be the smallest nonzero and largest singular values of the full rank matrix $B$, and suppose that $B\left(\begin{array}{l}Y Z \\ Y\end{array}\right)=\left(\begin{array}{ll}L & 0\end{array}\right)$, where $\left(\begin{array}{l}Y Z \\ Y\end{array}\right)$ is orthogonal and $L$ is nonsingular. Suppose further that $A$ has extreme eigenvalues $\lambda_{\min }^{A} \leq \lambda_{\max }^{A}, Z^{T} A Z$ is positive definite with extreme eigenvalues $0<\lambda_{\min }^{Z} \leq \lambda_{\max }^{Z}$ and that $Y^{T} A Y$ has extreme eigenvalues $\lambda_{\min }^{Y} \leq \lambda_{\max }^{Y}$. Then the eigenvalues $\mu$ of $\mathcal{M}$ are contained in

$$
\begin{aligned}
& {\left[\frac{1}{2}\left(\lambda_{\min }^{A}-\sqrt{\left(\lambda_{\min }^{A}\right)^{2}+4 \sigma_{\max }^{2}}\right), \frac{1}{2}\left(\lambda_{\max }^{Y}-\sqrt{\left(\lambda_{\max }^{Y}\right)^{2}+4 \sigma_{\min }^{2}}\right)\right] } \\
\cup & {\left[\frac{1}{2}\left(\lambda_{\min }^{Y}+\sqrt{\left(\lambda_{\min }^{Y}\right)^{2}+4 \sigma_{\min }^{2}}\right), \frac{1}{2}\left(\lambda_{\max }^{A}+\sqrt{\left(\lambda_{\max }^{A}\right)^{2}+4 \sigma_{\max }^{2}}\right)\right] } \\
\cup & {\left[\gamma, \lambda_{\max }^{Z}\right] . }
\end{aligned}
$$

where $\gamma<\lambda_{\min }^{Z}$ is the smallest positive root of the cubic equation

$$
\mu^{3}-\mu^{2}\left(\lambda_{\min }^{Z}+\lambda_{\min }^{A}\right)+\mu\left(\lambda_{\min }^{Z} \lambda_{\min }^{A}-\|A\|^{2}-\sigma_{\min }^{2}\right)+\lambda_{\min }^{Z} \sigma_{\min }^{2}=0 .
$$

Proof. Let $[z ; y ; x]$ be an eigenvector corresponding to an eigenvalue $\mu$ of $(2.9)$. Then

$$
\begin{aligned}
Z^{T} A Z z+Z^{T} A Y y & =\mu z \\
Y^{T} A Z z+Y^{T} A Y y+L^{T} x & =\mu y \\
\text { and } L y & =\mu x .
\end{aligned}
$$

Since $\mathcal{M}$ is nonsingular, $\mu \neq 0$, and hence

$$
Y^{T} A Z z+Y^{T} A Y y+L^{T} L y / \mu=\mu y
$$

from (2.11b) and (2.11c). Hence forming the inner products of (2.11a) with $z$ and (2.12) with $y$, we find

$$
\begin{aligned}
z^{T} Z^{T} A Z z+z^{T} Z^{T} A Y y & =\mu\|z\|^{2} \\
\text { and } y^{T} Y^{T} A Z z+y^{T} Y^{T} A Y y+\|L y\|^{2} / \mu & =\mu\|y\|^{2} .
\end{aligned}
$$

Subtracting (2.13) from (2.14) gives

$$
y^{T} Y^{T} A Y y+\|L y\|^{2} / \mu-\mu\|y\|^{2}=z^{T} Z^{T} A Z z-\mu\|z\|^{2} .
$$

Now suppose that $\mu \geq \lambda_{\max }^{\mathrm{z}}>0$. In this case (2.15) implies that

$$
\left(\sigma_{\min }^{2}+\lambda_{\min }^{\mathrm{Y}} \mu-\mu^{2}\right)\|y\|^{2} \leq\|L y\|^{2}+\mu y^{T} Y^{T} A Y y-\mu^{2}\|y\|^{2} \leq 0
$$

in which case

$$
\mu \geq \frac{1}{2}\left(\lambda_{\min }^{\mathrm{Y}}+\sqrt{\left(\lambda_{\min }^{\mathrm{Y}}\right)^{2}+4 \sigma_{\min }^{2}}\right) .
$$

By contrast, if $\mu \leq 0 \leq \lambda_{\min }^{z},(2.15)$ implies that

$$
\|L y\|^{2} / \mu+y^{T} Y^{T} A Y y-\mu\|y\|^{2} \geq 0
$$


in which case

$$
\left(\sigma_{\min }^{2}+\lambda_{\max }^{\mathrm{Y}} \mu-\mu^{2}\right)\|y\|^{2} \leq\|L y\|^{2}+\mu y^{T} Y^{T} A Y y-\mu^{2}\|y\|^{2} \leq 0
$$

and thus

$$
\mu \leq \frac{1}{2}\left(\lambda_{\max }^{\mathrm{Y}}-\sqrt{\left(\lambda_{\max }^{\mathrm{Y}}\right)^{2}+4 \sigma_{\min }^{2}}\right) .
$$

The remaining interval bounds follow from Proposition 2.2.

\subsection{Augmenting the $(1,1)$ block}

A useful alternative to (1.4) is to note that the solution also satisfies the augmented system

$$
\left[\begin{array}{cc}
A+\tau B^{T} B & B^{T} \\
B & 0
\end{array}\right]\left[\begin{array}{l}
x \\
y
\end{array}\right]=\left[\begin{array}{c}
a+\tau B^{T} b \\
b
\end{array}\right]
$$

for any scalar $\tau[8]$. Thus the eigenvalues of

$$
\mathcal{M}(\tau)=\left[\begin{array}{cc}
A+\tau B^{T} B & B^{T} \\
B & 0
\end{array}\right]
$$

may be of interest. It is well known [1, Cor.12.9] that $A$ is positive definite on the kernel of $B$ if and only if $A+\tau B^{T} B$ is positive definite for all sufficiently large $\tau$, and hence saddle-point methods appropriate when the $(1,1)$ block is definite may be applied. We now estimate how large $\tau$ needs be for this to happen.

Lemma 2.10. Let $\sigma_{\min }$ be the smallest nonzero singular value of the full rank matrix

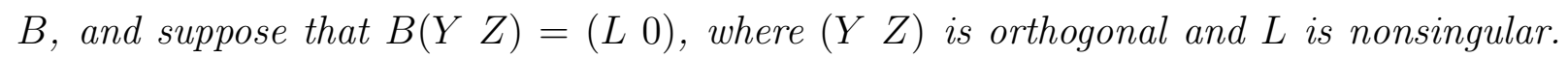
Suppose further that $A$ is positive definite on the kernel of $B$, so that the leftmost eigenvalue of $Z^{T} A Z, \lambda_{\min }^{Z}>0$, and the leftmost eigenvalue of $Y^{T} A Y$ be $\lambda_{\min }^{Y}$. Then the matrix $A+\tau B^{T} B$ is positive definite whenever

$$
\tau>\frac{1}{\sigma_{\min }^{2}}\left(\frac{\|A\|^{2}}{\lambda_{\min }^{Z}}-\lambda_{\min }^{Y}\right) .
$$

Proof. It follows immediately that $A+\tau B^{T} B$ is similar to the matrix

$$
\left[\begin{array}{cc}
Z^{T} A Z & Z^{T} A Y \\
Y^{T} A Z & Y^{T} A Y+\tau L L^{T}
\end{array}\right] .
$$

Therefore, $A+\tau B^{T} B$ is positive definite if and only if both $Z^{T} A Z$ and $Y^{T} A Y+\tau L L^{T}-$ $Y^{T} A Z\left(Z^{T} A Z\right)^{-1} Z^{T} A Y$ are positive definite. Since the first requirement is satisfied by assumption, we need only verify the second. For $w \neq 0$, we have

$$
\begin{aligned}
w^{T} Y^{T} A Y w+\tau w^{T} L L^{T} w & \geq\left(\lambda_{\min }^{\mathrm{Y}}+\tau \sigma_{\min }^{2}\right)\|w\|^{2}>\frac{\|A\|^{2}}{\lambda_{\min }^{\mathrm{Z}}}\|w\|^{2} \\
& \geq w^{T} Y^{T} A Z\left(Z^{T} A Z\right)^{-1} Z^{T} A Y w
\end{aligned}
$$

by assumption (2.16). 
Example 2.11. We consider once again Example 2.6, with $\|A\|=3, \lambda_{\min }^{\mathrm{Y}}=-0.01$, $\sigma_{\min }=3$ and $\lambda_{\min }^{z}=0.01$. The condition in Lemma 2.10 requires that

$$
\tau>\frac{1}{\sigma_{\min }^{2}}\left(\frac{\|A\|^{2}}{\lambda_{\min }^{\mathrm{Z}}}-\lambda_{\min }^{\mathrm{Y}}\right) \approx 100.002
$$

For $\tau=100$, which only barely fails to satisfy the condition, we obtain $\left.\sigma\left(A+\tau B^{T} B\right)\right)=$ $\left\{-1.1111 \cdot 10^{-7}, 900\right\}$. On the other hand, for $\left.\tau=101, \sigma\left(A+\tau B^{T} B\right)\right)=\left\{9.89 \cdot 10^{-5}, 909\right\}$, showing that our condition is sharp.

We can now derive bounds for the spectrum of $A+\tau B^{T} B$.

Proposition 2.12. Assume that the hypotheses of Lemma 2.10 hold, that $\tau$ satisfies (2.16) and that additionally the rightmost eigenvalues of $Z^{T} A Z$ and $Y^{T} A Y$ and largest singular value of $B$ are $\lambda_{\max }^{Z}, \lambda_{\max }^{Y}$ and $\sigma_{\max }$ respectively. Then the eigenvalues of $A+\tau B^{T} B$ are contained in the union of the positive intervals

$$
\begin{gathered}
{\left[\lambda_{\min }^{Z}, \lambda_{\max }^{Z}\right] \cup\left[\xi_{\min }, \xi_{\max }\right] \bigcup} \\
{\left[\frac { 1 } { 2 } \left(\xi_{\min }+\lambda_{\min }^{Z}-\sqrt{\left(\xi_{\min }-\lambda_{\min }^{Z}\right)^{2}+4\|A\|,} \frac{1}{2}\left(\xi_{\min }+\lambda_{\min }^{Z}+\sqrt{\left(\xi_{\min }-\lambda_{\min }^{Z}\right)^{2}+4\|A\|}\right] \cup\right.\right.} \\
{\left[\frac { 1 } { 2 } \left(\xi_{\max }+\lambda_{\max }^{Z}-\sqrt{\left(\xi_{\max }-\lambda_{\max }^{Z}\right)^{2}+4\|A\|}, \frac{1}{2}\left(\xi_{\max }+\lambda_{\max }^{Z}+\sqrt{\left(\xi_{\max }-\lambda_{\max }^{Z}\right)^{2}+4\|A\|}\right],\right.\right.}
\end{gathered}
$$

where $\xi_{\min }=\lambda_{\min }^{Y}+\tau \sigma_{\min }^{2}$ and $\xi_{\max }=\lambda_{\max }^{Y}+\tau \sigma_{\max }^{2}$.

Proof. Let $\lambda$ be an eigenvalue of $(2.17)$ and let $[x ; y]$ be the corresponding eigenvector. Then

$$
Z^{T} A Z x+Z^{T} A Y y=\lambda x \text { and } Y^{T} A Z x+\left(Y^{T} A Y+\tau L L^{T}\right) y=\lambda y .
$$

If $y=0$ then $\lambda$ is an eigenvalue of $Z^{T} A Z$, and thus $\lambda_{\min }^{\mathrm{z}} \leq \lambda \leq \lambda_{\max }^{\mathrm{Z}}$, which is the first eigenvalue interval. Similarly, if $x=0$ then $\lambda$ is an eigenvalue of $Y^{T} A Y+\tau L L^{T}$. In this case it follows immediately from (2.18) and [9, Thm.8.1.5] that

$$
\xi_{\min }\|w\|^{2} \leq w^{T}\left(Y^{T} A Y+\tau L L^{T}\right) w \leq \xi_{\max }\|w\|^{2},
$$

which provides the second eigenvalue interval.

Assume now that $0<\lambda<\lambda_{\min }^{Z}$, so that $Z^{T} A Z-\lambda I$ is positive definite. Therefore, from the first equation in (2.19) we obtain $x=-\left(Z^{T} A Z-\lambda I\right)^{-1} Z^{T} A Y y$ which, on substitution into the second, yields

$$
\left(Y^{T} A Y+\tau L L^{T}\right) y-Y^{T} A Z\left(Z^{T} A Z-\lambda I\right)^{-1} Z^{T} A Y y=\lambda y .
$$

Multiplying from the left by $0 \neq y^{T}$, we obtain

$$
\xi_{\min } \leq \lambda+\left(\lambda_{\min }^{\mathrm{z}}-\lambda\right)^{-1}\|A\|^{2}, \quad \text { that is } \quad \lambda^{2}-\lambda\left(\xi_{\min }+\lambda_{\min }^{\mathrm{z}}\right)+\xi_{\min } \lambda_{\min }^{\mathrm{z}}-\|A\|^{2} \leq 0 .
$$

The extremes of the third spectral interval follow from this last inequality, and by noticing that $\xi_{\min } \lambda_{\min }^{Z}-\|A\|^{2}>0$ because of (2.16). 
Finally, assume that $\lambda>\lambda_{\max }^{\mathrm{z}}$, so that $Z^{T} A Z-\lambda I$ is negative definite. As before $(2.20)$ holds, but now gives

$\lambda \leq \xi_{\max }+\left(\lambda-\lambda_{\max }^{\mathrm{z}}\right)^{-1}\|A\|^{2}, \quad$ that is $\lambda^{2}-\lambda\left(\xi_{\max }+\lambda_{\max }^{\mathrm{z}}\right)+\xi_{\max } \lambda_{\max }^{\mathrm{z}}-\|A\|^{2} \leq 0$.

Since $\xi_{\max } \lambda_{\max }^{\mathrm{Z}}-\|A\|^{2} \geq \xi_{\min } \lambda_{\min }^{\mathrm{Z}}-\|A\|^{2}>0$, it follows that $\lambda$ lies in the last of our eigenvalue intervals.

Armed with these bounds, it is trivial (but not especially edifying) to obtain eigenvalue interval bounds for $\mathcal{M}(\tau)$ for $\tau$ satisfying (2.16) using Proposition 2.3; simply replace $\lambda_{\min }^{\mathrm{A}}$ and $\lambda_{\max }^{\mathrm{A}}$ with the smallest and largest interval bounds from Proposition 2.12. More sophisticated bounds may be obtained from Propositions 2.8 and 2.9 if in addition we replace $\lambda_{\min }^{\mathrm{Y}}$ and $\lambda_{\max }^{\mathrm{Y}}$ by $\xi_{\min }$ and $\xi_{\max }$. All of these illustrate, as one might expect, the separation of the eigenvalue intervals into sub-intervals which grow asymptotically linearly with $\tau$ and those which are independent of $\tau$, and thus the worsening conditioning if $\tau$ is picked too large.

\section{Non-zero $(2,2)$ blocks}

We now turn our attention to the "stabilized" saddle-point matrix

$$
\mathcal{M}_{\mathcal{C}}=\left[\begin{array}{cc}
A & B^{T} \\
B & -C
\end{array}\right]
$$

where we assume that $C$ is symmetric and positive semi-definite. In the case where $A$ is positive definite the following result is known.

Proposition 3.1. [16, Lem.2.2] Assume that $A$ is positive definite, let $\lambda_{\min }^{A}, \lambda_{\max }^{A}$ be the smallest (positive) and largest (positive) eigenvalues of $A$, let $\sigma_{\min }, \sigma_{\max }$ be the smallest nonzero and largest singular values of the full rank matrix $B$, and let $C$ be positive semidefinite with largest eigenvalue $\lambda_{\max }^{C}$. Then the eigenvalues $\mu$ of $\mathcal{M}_{\mathcal{C}}$ are contained in $\mathcal{I}^{-} \cup \mathcal{I}^{+} \subset \mathbb{R}$ with

$$
\begin{aligned}
& \mathcal{I}^{-}=\left[\frac{1}{2}\left(\lambda_{\min }^{A}-\lambda_{\max }^{C}-\sqrt{\left(\lambda_{\min }^{A}+\lambda_{\max }^{C}\right)^{2}+4 \sigma_{\max }^{2}}\right), \frac{1}{2}\left(\lambda_{\max }^{A}-\sqrt{\left(\lambda_{\max }^{A}\right)^{2}+4 \sigma_{\min }^{2}}\right)\right] \subset \mathbb{R}^{-} \\
& \mathcal{I}^{+}=\left[\lambda_{\min }^{A}, \frac{1}{2}\left(\lambda_{\max }^{A}+\sqrt{\left(\lambda_{\max }^{A}\right)^{2}+4 \sigma_{\max }^{2}}\right)\right] \subset \mathbb{R}^{+} .
\end{aligned}
$$

For the more general case when $A$ is indefinite, care is needed. In particular a naive application of the well-know eigenvalue perturbation lemma gives the following result.

Proposition 3.2. Assume that $A$ is positive definite on the kernel of $B$, so that the leftmost eigenvalue of $Z^{T} A Z, \lambda_{\min }^{Z}>0$. Let $\lambda_{\min }^{A}, \lambda_{\max }^{A}$ be the leftmost (perhaps negative) and rightmost eigenvalues of $A$, let $\sigma_{\min }, \sigma_{\max }$ be the smallest nonzero and largest singular 
values of the full rank matrix $B$, and let $C$ be positive semi-definite with largest eigenvalue $\lambda_{\max }^{C}$. Furthermore let $\gamma<\lambda_{\min }^{Z}$ be the smallest positive root of the cubic equation

$$
\mu^{3}-\mu^{2}\left(\lambda_{\min }^{Z}+\lambda_{\min }^{A}\right)+\mu\left(\lambda_{\min }^{Z} \lambda_{\min }^{A}-\|A\|^{2}-\sigma_{\min }^{2}\right)+\lambda_{\min }^{Z} \sigma_{\min }^{2}=0,
$$

and suppose that $\gamma>\lambda_{\max }^{C}$. Then the eigenvalues $\mu$ of $\mathcal{M}_{\mathcal{C}}$ are contained in $\mathcal{I}^{-} \cup \mathcal{I}^{+} \subset \mathbb{R}$ with

$$
\begin{aligned}
& \mathcal{I}^{-}=\left[\frac{1}{2}\left(\lambda_{\text {min }}^{A}-\sqrt{\left(\lambda_{\text {min }}^{A}\right)^{2}+4 \sigma_{\text {max }}^{2}}\right)-\lambda_{\text {max }}^{C}, \frac{1}{2}\left(\lambda_{\text {max }}^{A}-\sqrt{\left(\lambda_{\text {max }}^{A}\right)^{2}+4 \sigma_{\text {min }}^{2}}\right)\right] \subset \mathbb{R}^{-} \\
& \mathcal{I}^{+}=\left[\gamma-\lambda_{\text {max }}^{C}, \frac{1}{2}\left(\lambda_{\text {max }}^{A}+\sqrt{\left(\lambda_{\text {max }}^{A}\right)^{2}+4 \sigma_{\text {max }}^{2}}\right)\right] \subset \mathbb{R}^{+}
\end{aligned}
$$

Proof. This follows immediately by applying, e.g., [9, Thm.8.1.5] to the results in Proposition 2.2.

Note here the requirement $\gamma>\lambda_{\max }^{\mathrm{C}}$ which is needed to ensure that the eigenvalue intervals do not include the origin. Although this bound is most-likely pessimistic, it is clear that some bound on the size of $C$ relative to $A$ and $B$ is needed to prevent singularity; for instance the 2 by 2 matrix formed when $A=-1=C, B=1$ is singular.

In the optimization context as we have seen, if $C$ is non-singular, it is realistic to expect that $A+B^{T} C^{-1} B$ will be positive definite, and that its smallest eigenvalue will be bounded away from zero [12]. As a consequence, necessarily we will have that $A$ will be positive definite on the null space of $B$, but this may be far from sufficient.

To improve upon Proposition 3.2, we have the following result.

Proposition 3.3. Assume that $A$ is positive definite on the kernel of $B$, so that the leftmost eigenvalue of $Z^{T} A Z, \lambda_{\min }^{Z}>0$. Let $\lambda_{\min }^{A}, \lambda_{\max }^{A}$ be the leftmost (perhaps negative) and rightmost eigenvalues of $A$, let $\sigma_{\min }, \sigma_{\max }$ be the smallest nonzero and largest singular values of the full rank matrix $B$, and let $C$ be positive semi-definite with largest eigenvalue $\lambda_{\max }^{C}$. Suppose furthermore that

$$
\lambda_{\max }^{C}<\frac{\lambda_{\min }^{Z} \sigma_{\min }^{2}}{\|A\|^{2}-\lambda_{\min }^{A} \lambda_{\min }^{Z}} .
$$

Then the eigenvalues $\mu$ of $\mathcal{M}_{\mathcal{C}}$ are contained in $\mathcal{I}^{-} \cup \mathcal{I}^{+} \subset \mathbb{R}$ with

$$
\begin{aligned}
& \mathcal{I}^{-}=\left[\frac{1}{2}\left(\lambda_{\min }^{A}-\lambda_{\max }^{C}-\sqrt{\left(\lambda_{\min }^{A}+\lambda_{\max }^{C}\right)^{2}+4 \sigma_{\max }^{2}}\right), \frac{1}{2}\left(\lambda_{\max }^{A}-\sqrt{\left(\lambda_{\max }^{A}\right)^{2}+4 \sigma_{\min }^{2}}\right)\right] \subset \mathbb{R}^{-} \\
& \mathcal{I}^{+}=\left[\gamma_{C}, \frac{1}{2}\left(\lambda_{\max }^{A}+\sqrt{\left(\lambda_{\max }^{A}\right)^{2}+4 \sigma_{\max }^{2}}\right)\right] \subset \mathbb{R}^{+},
\end{aligned}
$$

where $\gamma_{C}<\lambda_{\min }^{Z}$ is the smallest positive root of the cubic equation

$$
\begin{gathered}
\mu^{3}-\mu^{2}\left(\lambda_{\min }^{Z}+\lambda_{\min }^{A}-\lambda_{\max }^{C}\right)-\mu\left(\|A\|^{2}-\lambda_{\min }^{A} \lambda_{\min }^{Z}+\lambda_{\max }^{C} \lambda_{\min }^{Z}+\lambda_{\max }^{C} \lambda_{\min }^{A}+\sigma_{\min }^{2}\right) \\
+\lambda_{\max }^{C} \lambda_{\min }^{A} \lambda_{\min }^{Z}-\lambda_{\max }^{C}\|A\|^{2}+\lambda_{\min }^{Z} \sigma_{\min }^{2}=0 .
\end{gathered}
$$


In particular,

$$
\gamma_{C} \geq \begin{cases}\frac{\lambda_{\max }^{C} \lambda_{\min }^{A} \lambda_{\min }^{Z}-\lambda_{\max }^{C}\|A\|^{2}+\lambda_{\min }^{Z} \sigma_{\min }^{2}}{-\lambda_{\min }^{Z} \lambda_{\min }^{A}+\|A\|^{2}+\sigma_{\min }^{2}+\left(\lambda_{\max }^{C}\right)^{2}} \quad \text { if } \lambda_{\min }^{Z}+\lambda_{\min }^{A}-\lambda_{\max }^{C} \leq 0 \\ \chi+\sqrt{\chi^{2}+\frac{-\lambda_{\max }^{C}\|A\|^{2}+\lambda_{\max }^{C} \lambda_{\min }^{A} \lambda_{\min }^{Z}+\lambda_{\min }^{Z} \sigma_{\min }^{2}}{\lambda_{\min }^{Z}+\lambda_{\min }^{A}-\lambda_{\max }^{C}}} & \text { otherwise, }\end{cases}
$$

where

$$
\chi=\frac{\lambda_{\min }^{Z} \lambda_{\min }^{A}-\|A\|^{2}-\sigma_{\min }^{2}-\lambda_{\max }^{C}\left(\lambda_{\min }^{Z}+\lambda_{\min }^{A}\right)}{2\left(\lambda_{\min }^{Z}+\lambda_{\min }^{A}-\lambda_{\max }^{C}\right)} .
$$

If $m=n$, we have instead

$$
\mathcal{I}^{+}=\left[\frac{1}{2}\left(\lambda_{\min }^{A}-\lambda_{\max }^{C}+\sqrt{\left(\lambda_{\min }^{A}+\lambda_{\max }^{C}\right)^{2}+4 \sigma_{\min }^{2}}\right), \frac{1}{2}\left(\lambda_{\max }^{A}+\sqrt{\left(\lambda_{\max }^{A}\right)^{2}+4 \sigma_{\max }^{2}}\right)\right] \subset \mathbb{R}^{+} .
$$

Proof. The right bound on each interval follows as before by applying, e.g., [9, Thm.8.1.5] to the results in Proposition 2.2. It remains to establish the left bounds. As before, let $[x ; y]$ be an eigenvector corresponding to an eigenvalue $\mu$. Thus

$$
\begin{aligned}
A x+B^{T} y & =\mu x \\
\text { and } B x & =(C+\mu I) y .
\end{aligned}
$$

We first consider the case $\mu<0$. Suppose that $\mu<\lambda_{\text {min }}^{\mathrm{A}}$. In this case, $A-\mu I$ is positive definite, and $\left\|(A-\mu I)^{-1}\right\| \leq 1 /\left(\lambda_{\min }^{\mathrm{A}}-\mu\right)$. Furthermore $y \neq 0$ as otherwise $(3.22 \mathrm{~b})$ and the positive definiteness of $A-\mu I$ would imply that both $x$ and $y$ are zero. Hence (3.22) and the stated assumptions give

$$
-\mu\|y\|^{2}=y^{T} C y+y^{T} B(A-\mu I)^{-1} B^{T} y \leq \lambda_{\max }^{\mathrm{C}}\|y\|^{2}+\sigma_{\max }^{2}\|y\|^{2} /\left(\lambda_{\min }^{\mathrm{A}}-\mu\right)
$$

which implies

$$
\mu \geq \frac{1}{2}\left(\lambda_{\min }^{\mathrm{A}}-\lambda_{\max }^{\mathrm{C}}-\sqrt{\left(\lambda_{\min }^{\mathrm{A}}+\lambda_{\max }^{\mathrm{C}}\right)^{2}+4 \sigma_{\max }^{2}}\right)
$$

Otherwise

$$
\mu \geq \lambda_{\min }^{\mathrm{A}} \geq \frac{1}{2}\left(\lambda_{\min }^{\mathrm{A}}-\lambda_{\max }^{\mathrm{C}}-\sqrt{\left(\lambda_{\min }^{\mathrm{A}}+\lambda_{\max }^{\mathrm{C}}\right)^{2}+4 \sigma_{\max }^{2}}\right)
$$

and the combination of the two inequalities gives the lower bound on $\mathcal{I}^{-}$.

Now suppose that $0<\mu<\lambda_{\text {min }}^{\mathrm{z}}$, so that $C+\mu I$ is positive definite. For $y=0$ it follows that $\eta$ is an eigenvalue of $Z^{T} A Z$. For $y \neq 0$, we thus have $B x \neq 0$. Substituting $y \neq 0$ from (3.22b) into (3.22a) yields

$$
A x+B^{T}(C+\mu I)^{-1} B x=\mu x .
$$


We write $x=x_{0}+x_{1}$ as in the proof of Proposition 2.2 where $x_{1} \neq 0$, and we recall that for $0<\mu<\lambda_{\text {min }}^{\mathrm{z}}$ it holds that $-x_{1}^{T} A x_{0} \leq\|A\|^{2}\left\|x_{1}\right\|^{2} /\left(\lambda_{\text {min }}^{\mathrm{z}}-\mu\right)$. We multiply the equation in (3.23) by $x_{1}^{T}$ and obtain

$$
\begin{aligned}
0 & =-x_{1}^{T} A x-x_{1}^{T} B^{T}(C+\mu I)^{-1} B x_{1}+\mu\left\|x_{1}\right\|^{2} \\
& \leq\left(-\lambda_{\min }^{\mathrm{A}}+\frac{\|A\|^{2}}{\lambda_{\min }^{\mathrm{Z}}-\mu}-\frac{\sigma_{\min }^{2}}{\mu+\lambda_{\max }^{\mathrm{C}}}+\mu\right)\left\|x_{1}\right\|^{2} .
\end{aligned}
$$

Since $\lambda_{\min }^{\mathrm{Z}}-\mu$ and $\mu+\lambda_{\max }^{\mathrm{C}}$ are both positive and $x_{1} \neq 0$, we can write

$$
\begin{aligned}
0 \leq-\mu^{3} & +\mu^{2}\left(\lambda_{\min }^{\mathrm{Z}}+\lambda_{\min }^{\mathrm{A}}-\lambda_{\max }^{\mathrm{C}}\right)+\mu\left(-\lambda_{\min }^{\mathrm{A}} \lambda_{\min }^{\mathrm{Z}}+\|A\|^{2}+\lambda_{\max }^{\mathrm{C}} \lambda_{\min }^{\mathrm{Z}}+\lambda_{\max }^{\mathrm{C}} \lambda_{\min }^{\mathrm{A}}\right. \\
& \left.+\sigma_{\min }^{2}\right)-\lambda_{\max }^{\mathrm{C}} \lambda_{\min }^{\mathrm{A}} \lambda_{\min }^{\mathrm{Z}}+\lambda_{\max }^{\mathrm{C}}\|A\|^{2}-\lambda_{\min }^{\mathrm{Z}} \sigma_{\min }^{2} \equiv-\phi^{\mathrm{C}}(\mu) .
\end{aligned}
$$

Note that $\phi^{\mathrm{C}}(0)=\lambda_{\max }^{\mathrm{C}} \lambda_{\min }^{\mathrm{A}} \lambda_{\min }^{\mathrm{z}}-\lambda_{\max }^{\mathrm{C}}\|A\|^{2}+\lambda_{\min }^{\mathrm{z}} \sigma_{\min }^{2}>0$ by assumption (3.21), while $\phi^{\mathrm{C}}\left(\lambda_{\text {min }}^{\mathrm{Z}}\right)=-\left(\lambda_{\min }^{\mathrm{Z}}+\lambda_{\max }^{\mathrm{C}}\right)\|A\|^{2}<0$. Thus the required bound $\gamma_{C}$ we seek is the smallest positive root of $\phi^{\mathrm{C}}(\mu)=0$ and $\gamma_{C}<\lambda_{\min }^{\mathrm{z}}$. Furthermore, since $\mu^{3}>0$ we may bound $-\phi^{\mathrm{C}}$ from above to give

$$
\begin{aligned}
& 0 \leq \mu^{2}(\left.\lambda_{\min }^{\mathrm{Z}}+\lambda_{\min }^{\mathrm{A}}-\lambda_{\max }^{\mathrm{C}}\right)+\mu\left(-\lambda_{\min }^{\mathrm{A}} \lambda_{\min }^{\mathrm{Z}}+\|A\|^{2}+\lambda_{\max }^{\mathrm{C}} \lambda_{\min }^{\mathrm{Z}}\right. \\
&\left.+\lambda_{\max }^{\mathrm{C}} \lambda_{\min }^{\mathrm{A}}+\sigma_{\min }^{2}\right)-\lambda_{\max }^{\mathrm{C}} \lambda_{\min }^{\mathrm{A}} \lambda_{\min }^{\mathrm{Z}}+\lambda_{\max }^{\mathrm{C}}\|A\|^{2}-\lambda_{\min }^{\mathrm{Z}} \sigma_{\min }^{2}
\end{aligned}
$$

and if $\lambda_{\text {min }}^{\mathrm{Z}}+\lambda_{\min }^{\mathrm{A}}-\lambda_{\max }^{\mathrm{C}}>0$ then $\gamma_{C}$ is obtained as the largest root of the equation associated with this quadratic inequality. If by contrast $\lambda_{\min }^{\mathrm{Z}}+\lambda_{\min }^{\mathrm{A}}-\lambda_{\max }^{\mathrm{C}} \leq 0,(3.24)$ gives

$$
\begin{gathered}
0 \leq \mu\left(-\lambda_{\min }^{\mathrm{A}} \lambda_{\min }^{\mathrm{Z}}+\|A\|^{2}+\lambda_{\max }^{\mathrm{C}} \lambda_{\min }^{\mathrm{Z}}+\lambda_{\max }^{\mathrm{C}} \lambda_{\min }^{\mathrm{A}}+\sigma_{\min }^{2}\right)-\lambda_{\max }^{\mathrm{C}} \lambda_{\min }^{\mathrm{A}} \lambda_{\min }^{\mathrm{Z}}+ \\
\lambda_{\max }^{\mathrm{C}}\|A\|^{2}-\lambda_{\min }^{\mathrm{Z}} \sigma_{\min }^{2},
\end{gathered}
$$

that is,

$\mu\left(-\lambda_{\min }^{\mathrm{A}} \lambda_{\min }^{\mathrm{Z}}+\|A\|^{2}+\lambda_{\max }^{\mathrm{C}}\left(\lambda_{\min }^{\mathrm{Z}}+\lambda_{\min }^{\mathrm{A}}\right)+\sigma_{\min }^{2}\right) \geq \lambda_{\max }^{\mathrm{C}}\left(\lambda_{\min }^{\mathrm{A}} \lambda_{\min }^{\mathrm{Z}}-\|A\|^{2}\right)+\lambda_{\min }^{\mathrm{Z}} \sigma_{\min }^{2}$.

Since $\lambda_{\min }^{\mathrm{Z}}+\lambda_{\min }^{\mathrm{A}} \leq \lambda_{\max }^{\mathrm{C}}$ we may write

$$
-\lambda_{\min }^{\mathrm{A}} \lambda_{\min }^{\mathrm{Z}}+\|A\|^{2}+\lambda_{\max }^{\mathrm{C}} \lambda_{\min }^{\mathrm{Z}}+\lambda_{\max }^{\mathrm{C}} \lambda_{\min }^{\mathrm{A}} \leq-\lambda_{\min }^{\mathrm{A}} \lambda_{\min }^{\mathrm{Z}}+\|A\|^{2}+\left(\lambda_{\max }^{\mathrm{C}}\right)^{2}
$$

where the last quantity is positive, and the bound on $\gamma_{C}$ thus follows.

If $m=n$ and $\mu>0,(3.23)$ and the stated assumptions give

$$
0=x^{T} A x+x^{T} B^{T}(C+\mu I)^{-1} B x-\mu\|x\|^{2} \geq\left(\lambda_{\min }^{\mathrm{A}}+\frac{\sigma_{\min }^{2}}{\lambda_{\max }^{\mathrm{C}}+\mu}-\mu\right)\|x\|^{2},
$$

which leads directly to the stated lower bound in this case.

Note that for $\lambda_{\max }^{\mathrm{C}}=0$ we recover the results from Proposition 2.2. 
Example 3.4. We consider once again the data from Example 2.7, and we set $C=$ $\operatorname{diag}(0.01,0.03)$. The condition of Proposition 3.3 is satisfied, as the largest eigenvalue of $C$ is less than $\frac{\lambda_{\min }^{Z} \sigma_{\min }^{2}}{\|A\|^{2}-\lambda_{\min }^{A} \lambda_{\min }^{Z}} \approx 0.07992 . \quad$ The eigenvalues of $\mathcal{M}_{C}$ are $\{-4.3537,-0.25122,0.21323,2.0,4.3517\}$ and the intervals of Proposition 3.3 are

$$
[-4.3544,-0.22974] \subset \mathbb{R}^{-}, \quad[0.047343,4.3528] \subset \mathbb{R}^{+},
$$

showing a very good agreement of three of the four extremes.

Remark 3.5. Consider the matrix

$$
\mathcal{M}_{C}=\left[\begin{array}{ccc}
\lambda_{\min }^{\mathrm{A}} & 0 & \sigma \\
0 & \lambda_{\max }^{\mathrm{A}} & 0 \\
\sigma & 0 & -\beta
\end{array}\right],
$$

with $\lambda_{\text {min }}^{\mathrm{A}}<0, \lambda_{\text {max }}^{\mathrm{A}}>0, \sigma>0$. If $\beta=-\sigma^{2} / \lambda_{\text {min }}^{\mathrm{A}}$ then $\mathcal{M}_{C}$ is singular.

Proof. It is readily seen that the eigenvalues of $\mathcal{M}_{C}$ are

$$
\mu_{1}=\lambda_{\max }^{\mathrm{A}}, \quad \mu_{2,3}=\lambda_{\min }^{\mathrm{A}}-\beta \pm \sqrt{\left(\beta+\lambda_{\min }^{\mathrm{A}}\right)^{2}+4 \sigma^{2}} .
$$

Substituting $\beta=-\sigma^{2} / \lambda_{\text {min }}^{\mathrm{A}}$ in the second expression we obtain $\mu_{2}=\lambda_{\text {min }}^{\mathrm{A}}+\sigma^{2} / \lambda_{\min }^{\mathrm{A}}+$ $\left|\lambda_{\min }^{\mathrm{A}}+\sigma^{2} / \lambda_{\text {min }}^{\mathrm{A}}\right|=0$.

In our context, the remark above shows that a condition on $\beta$, that here plays the role of the $(2,2)$ block, is required to maintain nonsingularity. It is then remarkable that for the example in the remark above, if $\lambda_{\min }^{\mathrm{Z}}=\lambda_{\max }^{\mathrm{A}}=\|A\|=-\lambda_{\min }^{\mathrm{A}}$, Proposition 3.2 requires that

$$
0 \leq \gamma_{\max }^{\mathrm{C}} \leq \frac{1}{2} \frac{-\sigma_{\min }^{2}}{\lambda_{\min }^{\mathrm{A}}},
$$

which is only half the value that would yield a singular matrix. For this reason, our condition on $\gamma_{\max }^{\mathrm{C}}$ seems to be reasonably sharp.

Example 3.6. We consider the matrices $A$ and $B$ from Example 2.6 for which the bound from the left extreme of $\mathcal{I}^{+}$of Proposition 2.2 was sharp. Now consider $C=8 \cdot 10^{-4}$. The eigenvalues of $\mathcal{M}_{C}$ are (exact to the first few decimal digits)

$$
\{-4.2454,0.00460,4.2400\}
$$

and $\|A\|=\left|\lambda_{\min }^{\mathrm{A}}\right| \approx 3+0.01^{2} / 6, \lambda_{\min }^{\mathrm{z}}=0.01, \sigma_{\min }=3$. We note that the value of $\lambda_{\max }^{\mathrm{C}}$ is significantly smaller than $\lambda_{\min }^{\mathrm{Z}} \sigma_{\min }^{2} /\left(\|A\|^{2}-\lambda_{\min }^{\mathrm{A}} \lambda_{\min }^{\mathrm{Z}}\right) \approx 0.00996$. Here $\lambda_{\min }^{\mathrm{Z}}+\lambda_{\min }^{\mathrm{A}}-\lambda_{\max }^{\mathrm{C}} \approx$ $-2.9908<0$ so that $\gamma_{C} \approx 0.00459098$, which is a very sharp lower bound of the smallest positive eigenvalue of $\mathcal{M}_{C}$. 
Recently Bai, Ng and Wang [2] have established qualitatively similar bounds for $\mathcal{M}_{\mathcal{C}}$ based on extreme eigenvalues of $B^{T} C^{-1} B$ and $A+B^{T} C^{-1} B$ rather than those of $A$ and of $Z^{T} A Z$, and the singular values of $B$. As here there is no assumption of definiteness of $A$. Significantly, in [2] the authors do not require that $B$ be of full rank; rather they require that $C$ be nonsingular. In the motivating (optimization) applications we are concerned with, the rightmost eigenvalues of $B^{T} C^{-1} B$ and $A+B^{T} C^{-1} B$ may be huge so that cancellation in the bounds involving these [2, Thm.2.1] may be significant. Nonetheless Bai, Ng and Wang's bounds provide a useful alternative to those we have established.

The bounds above for the spectrum of $\mathcal{M}_{C}$ may be specialized following the lines of those in $\S 2.1$. To limit the proliferation of cumbersome estimates we omit their explicit derivation.

The assumption that $B$ be of full rank in $\mathcal{M}_{C}$ is of course a limitation. In some cases, such as from the optimization application illustrated in (1.3), $B$ and $C$ are of the form

$$
B=\left[\begin{array}{l}
B_{1} \\
B_{2}
\end{array}\right] \text { and } C=\left[\begin{array}{cc}
C_{1} & 0 \\
0 & 0
\end{array}\right]
$$

with positive definite $C_{1}$. The natural assumption in this case is that $A+B_{1}^{T} C_{1}^{-1} B_{1}$ should be definite on the null space of the full-rank $B_{2}$. In this case,

$$
\mathcal{M}_{C}=\left[\begin{array}{cc}
\left(\begin{array}{cc}
A & B_{1}^{T} \\
B_{1} & -C_{1}
\end{array}\right) & \left(\begin{array}{c}
B_{2}^{T} \\
0
\end{array}\right) \\
\left(B_{2}\right. & 0
\end{array}\right) .
$$

This may then be interpreted as a matrix of the form $\mathcal{M}$ whose "structured" $(1,1)$ block is of the form $\mathcal{M}_{C}$. One can thus obtain eigenvalue intervals by applying Proposition 2.2 to the saddle point matrix using estimates for the eigenvalues of its structured $(1,1)$ block using, e.g., [2, Thm.2.1].

Unfortunately there are examples [14] for which the relationship between rank-deficient $B$ and singular $C$ is less explicit, at least from an algebraic viewpoint. If $A$ and $C+B B^{T}$ are positive definite, it is still possible to compute useful eigenvalue intervals for $\mathcal{M}_{C}[14$, Prop.3.1]. But when $A$ is indefinite, although one can write abstract conditions for the eigen-problem in terms of representations of the range space of $B$, they do not seem to us to be particularly illuminating.

\section{A first look at preconditioning strategies}

The efficient solution of large saddle point linear systems involving $\mathcal{M}$ or $\mathcal{M}_{C}$ strongly calls for preconditioners. Ideally these should take into account both the nature of the problem and its algebraic structure. Our setting is particularly challenging because of the indefiniteness of $A$. For simplicity, we only consider symmetric block diagonal preconditioners which take the form

$$
\mathcal{P}=\left[\begin{array}{cc}
P_{A} & O \\
O & P_{C}
\end{array}\right]
$$


for some symmetric $P_{A}$ and $P_{C}$. In this section we present some preliminary results obtained when either $P_{A}$ and/or $P_{C}$ are ideal approximations to their target matrices. A more general analysis for a larger class of diagonal blocks is beyond the scope of this paper, and deserves a specialized study. In addition, because of the indefiniteness of $A$, other, not necessarily block diagonal, preconditioners may be more suitable; see, e.g., $[2,5,7]$. Hence, this will be the topic of future investigation.

\subsection{Indefinite block-diagonal preconditioners}

Here we restrict our attention to block diagonal preconditioners of the form

$$
\mathcal{P}_{ \pm}=\left[\begin{array}{cc}
\widetilde{A} & O \\
O & \pm \widetilde{S}
\end{array}\right]
$$

where $\widetilde{A} \approx A$ (in fact, we use $\widetilde{A}=A$ ), and $\widetilde{S} \approx C+B \widetilde{A}^{-1} B^{T}$.

The following result generalizes well known spectral properties of $\mathcal{P}$ for $C=0$ to the case of indefinite but nonsingular $A$. The proof is the same as in the definite case.

Proposition 4.1. The following results hold.

1. Let $\mathcal{P}_{+}=\operatorname{blkdiag}\left(A, B A^{-1} B^{T}\right)$. Then

$$
\sigma\left(\mathcal{P}_{+}^{-1} \mathcal{M}\right) \subset\left\{1, \frac{1}{2}(1+\sqrt{5}), \frac{1}{2}(1-\sqrt{5})\right\} \subset \mathbb{R}
$$

2. Let $\mathcal{P}_{-}=\operatorname{blkdiag}\left(A,-B A^{-1} B^{T}\right)$. Then

$$
\sigma\left(\mathcal{P}_{-}^{-1} \mathcal{M}\right) \subset\left\{1, \frac{1}{2}(1+i \sqrt{3}), \frac{1}{2}(1-i \sqrt{3})\right\} \subset \mathbb{C}^{+} .
$$

Similar although less clean results may be obtained for $C \neq 0$, as shown in the next proposition.

Proposition 4.2. Let $C$ be symmetric and positive semidefinite, and let $\theta$ be the finite eigenvalues of the pair $\left(C+B A^{-1} B^{T}, C\right)$. Then the following results hold.

1. Let $\mathcal{P}_{+}=\operatorname{blkdiag}\left(A, C+B A^{-1} B^{T}\right)$. Then

$$
\sigma\left(\mathcal{P}_{+}^{-1} \mathcal{M}\right) \subset\left\{1, \frac{1}{2}(1 \pm \sqrt{5}), \frac{1}{2 \theta}\left(\theta-1 \pm \sqrt{(1-\theta)^{2}+4 \theta^{2}}\right)\right\} \subset \mathbb{R} .
$$

2. Let $\mathcal{P}_{-}=\operatorname{blkdiag}\left(A,-C-B A^{-1} B^{T}\right)$. Then

$$
\sigma\left(\mathcal{P}_{-}^{-1} \mathcal{M}\right) \subset\left\{1, \frac{1}{2}(1+i \sqrt{3}), \frac{1}{2}(1-i \sqrt{3}), \frac{1}{2 \theta}\left(\theta+1 \pm \sqrt{(1+\theta)^{2}-4 \theta^{2}}\right)\right\} \subset \mathbb{C}^{+} .
$$


Proof. From the first equation in

$$
A x+B^{T} y=\mu A x, \quad B x-C y=\mu\left(C+B A^{-1} B^{T}\right) y,
$$

we obtain $(\mu-1) x=A^{-1} B^{T} y$. Either $\mu=1$ or $x=A^{-1} B^{T} y /(\mu-1)$. Substituting this into the second equation and collecting terms we obtain $\mu C y=\left(\mu-\mu^{2}+1\right)(C+$ $\left.B A^{-1} B^{T}\right) y$. For $y \neq 0$, either $C y=0$, so that $\left(\mu-\mu^{2}+1\right)=0$ (the Schur complement is nonsingular), from which the eigenvalues $(1 \pm \sqrt{5}) / 2$ follow, or we can write $(C+$ $\left.B A^{-1} B^{T}\right)=\theta C y$, with $\theta=\mu /\left(\mu-\mu^{2}+1\right)$, from which the remaining expressions for the eigenvalues follow.

In the second case, following the same steps we arrive at the equation $\mu C y=(-\mu+$ $\left.\mu^{2}+1\right)\left(C+B A^{-1} B^{T}\right) y$ and the conclusions follow with similar reasonings.

In the case when $C=0$, we next extend this setting to the case when $\widetilde{S}$ is only an approximation to the Schur complement $B A^{-1} B^{T}$. We were not able to obtain similarly explicit results for $C \neq 0$.

Proposition 4.3. Let $\mathcal{P}_{ \pm}=\operatorname{blkdiag}(A, \pm \widetilde{S})$ with $A, \widetilde{S}$ nonsingular. Then

$$
\sigma\left(\mathcal{P}_{ \pm}^{-1} \mathcal{M}\right) \subset\{1,(1+\sqrt{1+4 \xi}) / 2,(1-\sqrt{1+4 \xi}) / 2\} \subset \mathbb{C}
$$

where the $\xi$ 's are the (possibly complex) eigenvalues of the pair $\left(B A^{-1} B^{T}, \pm \widetilde{S}\right)$.

Proof. From the first equation in

$$
A x+B^{T} y=\mu A x, \quad B x= \pm \mu \widetilde{S} y,
$$

we obtain $(\mu-1) x=A^{-1} B^{T} y$. Either $\mu=1$ or $x=A^{-1} B^{T} y /(\mu-1)$. Substituting this latter expression in the second equation we obtain $B A^{-1} B^{T} y= \pm \mu(\mu-1) \widetilde{S} y$. Setting $\xi=\mu(\mu-1)$ the result follows.

The result above emphasizes that complex eigenvalues may arise, due to the generic indefinitess of the blocks in the preconditioner. This fact is even more dramatic when an approximation $\widetilde{A}$ is used in place of $A$.

In general, it thus follows that this indefinite block preconditioner cannot be applied with MINRES, but other possibly nonsymmetric solvers need be employed.

\subsection{Positive-definite block-diagonal preconditioners}

Since by assumption $A$ is indefinite, the preconditioners considered in $\S 4.1$ will not be definite. If we wish to precondition iterative methods like MINRES, we need to require that both $P_{A}$ and $P_{C}$ are definite. In this section we analyze such a situation, restricting our attention to the case where $C=0$. 
One possibility is to exploit the augmented structure analyzed in $§ 2.2$. For $\tau$ sufficiently large so as to guarantee that $A+\tau B^{T} B$ is positive definite, using for example (2.16), one can apply the block-diagonal preconditioner (4.26) with $P_{A} \approx P_{A}(\tau)=A+\tau B^{T} B$ and $P_{C} \approx P_{A}(\tau)=B\left(A+\tau B^{T} B\right)^{-1} B^{T}$ to either $\mathcal{M}$ or $\mathcal{M}(\tau)$.

To investigate this further, suppose that $\mathcal{P}(\tau)=\operatorname{blkdiag}\left(P_{A}(\tau), P_{C}(\tau)\right)$. In the ideal case where we try to precondition $\mathcal{M}(\tau)$ with $\mathcal{P}(\tau)$ we have the following simple result.

Proposition 4.4. $\mathcal{P}(\tau)^{-1} \mathcal{M}(\tau)$ has eigenvalues $1,(1+\sqrt{5}) / 2,(1-\sqrt{5}) / 2$ with multiplicity $n-m, m$ and $m$, respectively.

Proof. The result is simply a direct application of Proposition 4.1 when $A$ is replaced by $A+\tau B^{T} B$.

For the case where we try to precondition $\mathcal{M}$ with the ideal $\mathcal{P}(\tau)$, the result is more complicated.

Proposition 4.5. Suppose that $B(Y Z)=(L 0)$ is of full rank and that $Z^{T} A Z$ and $P_{A}(\tau)$ are positive definite. Then $\mathcal{P}(\tau)^{-1} \mathcal{M}$ has eigenvalues

i) 1, of multiplicity $n-m+\operatorname{Nullity}(A)$;

ii) -1 , of multiplicity $\operatorname{Nullity}(A)$;

iii) $\left(\mu_{i} \pm \sqrt{\mu_{i}^{2}+4}\right) / 2, i=1, \ldots, m-\operatorname{Nullity}(A)$, where $\mu_{i}=\omega_{i} /\left(\omega_{i}+\tau\right)$ and $\omega_{i}$ are the eigenvalues of $L^{-T}\left(Y^{T} A Y-Y^{T} A Z\left(Z^{T} A Z\right)^{-1} Z^{T} A Y\right) L^{-1}$.

Proof. This is based on results from [8, §2.1]. Specifically if $\lambda$ is an eigenvalue of $\mathcal{P}(\tau)^{-1} \mathcal{M}$ with eigenvector $[x ; y]$

$$
\left[\begin{array}{cc}
A & B^{T} \\
B & 0
\end{array}\right]\left[\begin{array}{l}
x \\
y
\end{array}\right]=\lambda\left[\begin{array}{cc}
P_{A}(\tau) & 0 \\
0 & B P_{A}^{-1}(\tau) B^{T}
\end{array}\right]\left[\begin{array}{l}
x \\
y
\end{array}\right]
$$

from which it follows that

$$
\left(\lambda^{2} I-\lambda K-Q\right) z=0
$$

where $z=P_{A}^{\frac{1}{2}}(\tau) x$,

$$
K=P_{A}^{-\frac{1}{2}}(\tau) A P_{A}^{-\frac{1}{2}}(\tau) \text { and } Q=P_{A}^{-\frac{1}{2}}(\tau) B^{T}\left(B P_{A}^{-1}(\tau) B^{T}\right)^{-1} B P_{A}^{-\frac{1}{2}}(\tau) .
$$

Clearly $Q$ is an orthogonal projection matrix of rank $m$. More significantly, it shares its eigenvectors with $K[8$, Lem.2.3] (and of course $I$ ). But $Q$ is annihilated by vectors $v$ in the null-space of $B$ while $K v=v$ for these (and only these) vectors [8, Lem.2.4(1)]. Hence (4.27) implies that $n-m$ of the required eigenvalues satisfy $\lambda^{2}-\lambda=0$ and are thus take the value 1 since $\mathcal{M}$ is non-singular because $B$ is of full rank and $Z^{T} A Z$ is positive definite. 
The remaining $2 m$ eigenvalues correspond to eigenvectors $w_{i}$ of $Q$ in the range-space of $B$ for which $Q w_{i}=w_{i}$, and thus $K w_{i}=\mu_{i} w_{i}$, where $\mu_{i}$ is one of the $m$ non-unit eigenvalues of $K$. It then follows from (4.27) that the remaining eigenvalues are the roots of

$$
\lambda^{2}-\mu_{i} \lambda-1=0
$$

But if $K w_{i}=\mu_{i} w_{i}$, it follows immediately that

$$
A u_{i}=\mu_{i}\left(A+\tau B^{T} B\right) u_{i}
$$

where $u_{i}=P_{A}^{-\frac{1}{2}}(\tau) w_{i}$. Moreover (4.29) and the non-singularity of $\mathcal{M}$ imply that $\mu_{i}=0$ if and only if $u_{i}$ lies in the null-space of $A$-for these (4.28) implies that a further $\operatorname{Nullity}(A)$ of the required eigenvalues have the value 1 , while there are $\operatorname{Nullity}(A)$ eigenvalues at -1 . Because of (4.29), the remaining nonzero, non-unit eigenvalues $\mu_{i}$ of $K$ satisfy

$$
A u_{i}=\frac{\tau \mu_{i}}{1-\mu_{i}} B^{T} B u_{i}
$$

But the generalised eigenvalue problem $A u=\omega B^{T} B u$ is equivalent to

$$
\left[\begin{array}{ll}
Y^{T} A Y & Y^{T} A Z \\
Z^{T} A Y & Z^{T} A Z
\end{array}\right]\left[\begin{array}{l}
s \\
t
\end{array}\right]=\omega\left[\begin{array}{cc}
L^{T} L & 0 \\
0 & 0
\end{array}\right]\left[\begin{array}{l}
s \\
t
\end{array}\right]
$$

where $s=Y^{T} u$ and $t=Z^{T} u$, and the result follows immediately on eliminating $t$.

Notice that as $\tau$ increases the eigenvalues of $\mathcal{P}(\tau)^{-1} \mathcal{M}$ cluster around the two values \pm 1 .

We also remark that for $\mathcal{P}(\tau)$ positive definite, spectral interval bounds may be obtained for the preconditioned matrix $\mathcal{P}(\tau)^{-\frac{1}{2}} \mathcal{M P}(\tau)^{-\frac{1}{2}}$, by applying the results of sections 2 and 2.1 to the preconditioned blocks.

Example 4.6. Consider the data, $A=Q D Q^{T}, D=\operatorname{diag}[-1 ; 0 ; 1 ; 2 ; 3 ; 4], Q=I-$ $2 v v^{T} / v^{T} v, v^{T}=[1,2,3,4,5,6]$ and

$$
B=\left[\begin{array}{cccccc}
1 & 0 & 0.01 & 0 & 0 & 0 \\
0 & 2 & 0 & 0.01 & 0 & 0
\end{array}\right]
$$

Thus $A$ is similar to $D$ (and hence singular with nullity 1 ). Furthermore $A+\tau B^{T} B$ is positive definite for all $\tau$ larger than (roughly) 1.075. We see in Table 4.2 the predicted $n-m+\operatorname{Nullity}(A)=5$ eigenvalues at $1, \operatorname{Nullity}(A)=1$ eigenvalue at -1 and a remaining pair of eigenvalues which converge to \pm 1 as $\tau$ increases. 


\begin{tabular}{|c|cccccccc|}
\hline$\tau$ & \multicolumn{7}{|c|}{ eigenvalues } \\
\hline 1.075 & -2665.05 & -1.0 & $3.75 \cdot 10^{-4}$ & 1.0 & 1.0 & 1.0 & 1.0 & 1.0 \\
\hline 10.75 & -1.05707 & -1.0 & 0.94600 & 1.0 & 1.0 & 1.0 & 1.0 & 1.0 \\
\hline 107.5 & -1.00506 & -1.0 & 0.99496 & 1.0 & 1.0 & 1.0 & 1.0 & 1.0 \\
\hline 1075 & -1.00051 & -1.0 & 0.99950 & 1.0 & 1.0 & 1.0 & 1.0 & 1.0 \\
\hline
\end{tabular}

Table 4.2: Eigenvalues of $\mathcal{P}(\tau)^{-1} \mathcal{M}$ for increasing values of $\tau$.

\section{Conclusions}

We have generalised many of the known eigenvalue bounds for saddle-point and stabilized saddle-point matrices with a definite $(1,1)$ block to the indefinite case. We have given eigenvalue bounds for the augmented formulation, also giving a sufficient condition for the $(1,1)$ block to be definite. We have also provided eigenvalue estimates for a number of block preconditioners, although the cases considered are somewhat idealized in that they assume inversion of "exact" diagonal blocks. It remains to consider how best to approximate these blocks while retaining the excellent clustering predicted from the exact case.

\section{Acknowledgement}

We would like to express our gratitude to the organisers of the 2008 LMS Durham Symposium on Computational Linear Algebra for Partial Differential Equations, which proved to be the stimulus for this work.

\section{References}

[1] M. Avriel. Nonlinear Programming: Analysis and Methods. Prentice-Hall, Englewood Cliffs, New Jersey, 1976.

[2] Z.-Z. Bai, M. K. Ng, and Z.-Q. Wang. Constraint preconditioners for symmetric indefinite matrices. Manuscript, Department of Mathematics, Hong Kong Baptist University, 2008.

[3] M. Benzi, G. H. Golub, and J. Liesen. Numerical solution of saddle point problems. Acta Numerica, 14:1-137, 2005.

[4] Y. Chabrillac and J.-P. Crouzeix. Definiteness and semidefiniteness of quadratic forms revisited. Linear Algebra and its Applications, 63:283-292, 1984.

[5] H. S. Dollar, N. I. M. Gould, M. Stoll, and A. J. Wathen. A Bramble-Pasciaklike method with applications in optimization. Technical Report RAL-TR-2008-017, Rutherford Appleton Laboratory, Chilton, Oxfordshire, England, 2008. 
[6] H. C. Elman, D. J. Silvester, and A. J. Wathen. Finite-Elements and Fast Iterative Solvers: with applications in Incompressible Fluid Dynamics. Oxford University Press, Oxford, 2005, to appear.

[7] A. Forsgren, P. E. Gill, and J. D. Griffin. Iterative solution of augmented systems arising in interior methods. SIAM Journal on Optimization, 18(2):666-690, 2007.

[8] G. H. Golub, C. Greif, and J. M. Varah. An algebraic analysis of a block diagonal preconditioner for saddle point systems. SIAM Journal on Matrix Analysis and Applications, 27(3):779-792, 2006.

[9] G. H. Golub and C. F. Van Loan. Matrix computations. Johns Hopkins University Press, Baltimore, third edition, 1996.

[10] J. Gondzio and A. Grothey. Direct solution of linear systems of size $10^{9}$ arising in optimization with interior point methods. In R. Wyrzykowski, J. Dongarra, N. Meyer, and J. Wasniewski, editors, Parallel Processing and Applied Mathematics PPAM 2005, number 3911 in Lecture Notes in Computer Science, pages 513-525, Heidelberg, Berlin, New York, 2006. Springer Verlag.

[11] N. I. M. Gould. On practical conditions for the existence and uniqueness of solutions to the general equality quadratic-programming problem. Mathematical Programming, 32(1):90-99, 1985.

[12] N. I. M. Gould. On the accurate determination of search directions for simple differentiable penalty functions. IMA Journal of Numerical Analysis, 6:357-372, 1986.

[13] J. Nocedal and S. J. Wright. Numerical Optimization. Series in Operations Research. Springer Verlag, Heidelberg, Berlin, New York, second edition, 2006.

[14] I. Perugia and V. Simoncini. Block-diagonal and indefinite symmetric preconditioners for mixed finite element formulations. Numerical Linear Algebra with Applications, $7(7-8): 585-616,2000$.

[15] T. Rusten and R. Winther. A preconditioned iterative method for saddlepoint problems. SIAM Journal on Matrix Analysis and Applications, 13(3):897-904, 1992.

[16] D. J. Silvester and A. J. Wathen. Fast iterative solution of stabilised Stokes systems part II: Using general block preconditioners. SIAM Journal on Numerical Analysis, 31(5):1352-1367, 1994. 